\title{
EFFECTS OF DIAGNOSTIC ULTRASOUND ON SISTER CHROMATID EXCHANGE FREQUENCIES OF THE AMNIOTIC FLUID CELLS OF THE PREGNANT WOMEN
}

\author{
Ma Changuun, ${ }^{1}$ Chen Yuancha, ${ }^{\perp}$ Chen Sufang, ${ }^{1}$ \\ Huo Peidan, ${ }^{1}$ Luo AnXue, ${ }^{2}$ Zhou YaLI, ${ }^{2}$ \\ and Lin Youlin ${ }^{2}$ \\ ${ }^{1}$ Department of Histology, Embryology and Cytogenetics Family Planning \\ Research Institute of Sichuan Chengdu, Sichuan, \\ People's Republic of China \\ ${ }^{2}$ Gynecology and Obstetric Hospital Chengdu, Sichuan, \\ People's Republic of China
}

\begin{abstract}
Summary The diagnostic ultrasound was given to 21 healthy pregnant women who were undergoing induced abortion, and sister chromatid exchange (SCE) was analyzed on amniotic fluid cells before and after the ultrasonic exposure. There was no linear relationship between the SCE frequency and time of ultrasonic exposure. The SCE frequency was not significantly increased after the exposure as compared with that before the exposure. The data suggest that diagnostic ultrasound is a safe procedure when used for prenatal diagnosic.
\end{abstract}

\section{INTRODUCTION}

In the past few years, ultrasound has been increasingly used in prenatal diagnosis in various parts of the world. However, the possibility that ultrasound can induce gene mutation has been widely concerned by scientists. Haupt et al. (1981) designed a simple model of $37^{\circ} \mathrm{C}$ water bath that simulated the spatial and sonographic conditions of an exposed gravid abdomen and uterus. Cultured human lymphocytes were placed within the model and exposed to various intensities of ultrasound. The authors used this method to analyze sister chromatid exchange (SCE) of the human lymphocytes and found that significant differences both in the distribution and the means of SCE frequencies for control versus exposed cells. Therefore, they suggested that minimizing the number and length of exposure per patient would be prudent (Haupt et al., 1981). Two other studies evaluated the effect of ultrasound on SCE frequency in human lymphocytes with contradictory results (Morris and Palmer, 1978; Liebeskind et al., 1979). Lundberg et al. (1982) 
reported the result of a study of in vivo diagnostic ultrasound on sister chromatid exchange frequency in amniotic fluid cell. There were some shortcomings in their report. First, the cell growth from initiation to harvest was too long (usually 14 to 21 days). Second, in the second period of SCE 25 metaphases per case was not reached. Third, the control cases were much less than the experimental cases and the control case and experimental case were not the same subject. Since every pregnant woman has her own specific environment and constitutions, the comparability in their experiment was not so satisfactory. Moreover, the variables such as abdominal wall thickness, placental position, and fetal posture can not be controlled. The above factors would influence the accuracy of the experiment.

In view of the shortcomings and limitations of the above research, we think that further improvement of methodology and design is needed in such a research.

\section{MATERIALS AND METHODS}

The samples of amniotic fluid were obtained from 21 healthy pregnant women who were undergoing induced abortion within 12-24 weeks of pregnancy. These subjects had not been exposed to any known mutagens nor teratogens, and did not smoke. Before giving a diagnostic ultrasound and inducing an abortion, each subject's personal history and pedigree were requested. The 21 selected subjects were divided into seven groups, each consisting of three. The diagnostic ultrasound was given to the women from abdomen down to the uterus and amniotic cavity, and the length of exposure for each group was different, i.e., 3, 6, 9, 12, 15, 18 and $21 \mathrm{~min}$, respectively; before and after the exposure, $20 \mathrm{ml}$ amniotic fluid were drawn as controls and experiments. For ultrasonography, the model SDL-100A frequency, $3.5 \mathrm{MHZ}$, range $21 \mathrm{~cm}($ deep) $\times 8.5$ (wide) was used.

The amniotic fluid cells of both the controls and the experimentals were cultured under the same condition, using the same culture medium and the same procedure. Chromosomes were prepared at the same time. The method used for amniotic fluid cell culture was an improved method by our laboratory (Ma et al., 1985). The cell growth from initiation to harvest usually took 9 days, sometimes 12 days. BrdU was added to the culture medium to make the final concentration $1.25 \mu \mathrm{g} / \mathrm{ml}$, and then the culture was continued in the dark place for $40 \mathrm{hr}$. Four hours before the end of culture, colchicine was added to the medium at a final concentration of 0.25 $\mu \mathrm{g} / \mathrm{ml}$. Preparation of the slide and procedure of staining were based on the standard method (Ma et al., 1983). Twenty-five metaphases, each having the chromosome number $2 n=46$, were selected at random. Exchange in the terminal of sister chromatid was regarded as one SCE, in the middle of sister chromatid as two SCEs, and in the centromeric region as one SCE. 


\section{RESULTS AND DISCUSSION}

From the exposure time and SCE frequency we sought the linear regression equation, $\hat{y}=8.3276-0.0913 x$ (Fig. 1). According to the result of analysis of variance for linear regression equation (Table 1) $F_{2}=3.21(p>0.05), F_{1}=1.97(p>0.05)$, we concluded that there was no linear relationship between the SCE frequency and time of ultrasonic exposure. We compared the SCE of amniotic fluid cells before and after exposure for 21 pregnant women with the diagnostic ultrasound. There was no statistically significant difference between the SCE before and after exposure $(0.5>\mathrm{p}>0.2)$ (Table 2).

Liebeskind et al. (1979) found that the frequency of sister chromatid exchanges increased in freshly isolated human lymphocytes as well as in a continuously growing lymphoblast line by exposure to diagnostic level of ultrasound for $30 \mathrm{~min}$. So they believed that most clinical examination of the fetus are of short duration and the

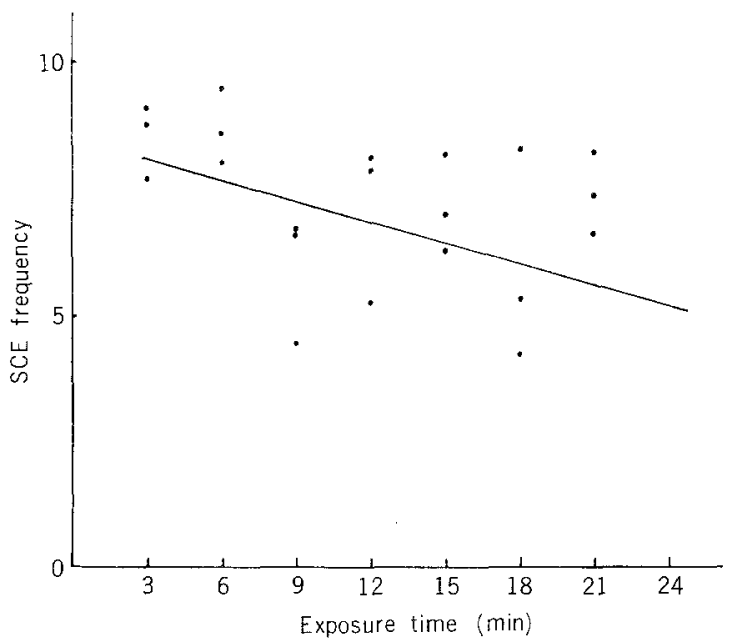

Fig. 1. The regression line of SCE frequency $(y)$ on exposure time $(x)$ to ultrasound in vivo.

Table 1. The analysis of variance for linear regression equation between exposure time of diagnostic ultrasound and SCE of amniotic fluid cells.

\begin{tabular}{lrrrrr}
\hline Source of variation & \multicolumn{1}{c}{ SS } & $\mu$ & MS & F & p \\
\hline Total & 43.58 & 20 & & & \\
Due to regression & 6.29 & 1 & 6.29 & $F_{2}=3.21$ & $>0.05$ \\
Misfitting & 15.40 & 5 & 3.08 & $F_{1}=1.97$ & $>0.05$ \\
Residue & 21.88 & 14 & 1.56 & & \\
\hline
\end{tabular}


Table 2. The paired data analysis of SCE in diagnostic ultrasound before and after exposure.

\begin{tabular}{|c|c|c|c|}
\hline $\begin{array}{l}\text { No. of the } \\
\text { pregnant women }\end{array}$ & Before exposure & After exposure & Exposure time \\
\hline 1 & 8.48 & 8.76 & 3 \\
\hline 2 & 7.88 & 9.04 & 3 \\
\hline 3 & 7.60 & 7.72 & 3 \\
\hline 4 & 7.80 & 8.00 & 6 \\
\hline 5 & 5.56 & 8.60 & 6 \\
\hline 6 & 6.28 & 9.48 & 6 \\
\hline 7 & 5.80 & 4.44 & 9 \\
\hline 8 & 8.32 & 6.68 & 9 \\
\hline 9 & 9.92 & 6.64 & 9 \\
\hline 10 & 5.20 & 5.24 & 12 \\
\hline 11 & 9.04 & 8.08 & 12 \\
\hline 12 & 7.68 & 7.84 & 12 \\
\hline 13 & 9.44 & 6.28 & 15 \\
\hline 14 & 7.96 & 8.12 & 15 \\
\hline 15 & 7.28 & 7.00 & 15 \\
\hline 16 & 6.88 & 5.32 & 18 \\
\hline 17 & 7.84 & 8.24 & 18 \\
\hline 18 & 4. 60 & 4. 24 & 18 \\
\hline 19 & 8.96 & 7.36 & 21 \\
\hline 20 & 6.72 & 6.60 & 21 \\
\hline 21 & 8.80 & 8.20 & 21 \\
\hline
\end{tabular}

surrounding maternal tissues attenuate the ultrasonic beam. However, their findings suggest that ultrasound may not be entirely innocuous. Haupt et al. (1981) reported the ultrasonic induction of sister chromatid exchanges in human lymphocytes. Although the cultures in their study were derived from adult lymphocytes rather than fetal tissues, they believed that fetal cells could show different responses, probably because of their actively dividing state. This problem is worth notice. It is generally believed that power of diagnostic ultrasound has no harm to human body, but whether it may induce a delicate fetal cell gene micromutation is still a question.

Our SCE research indicates that the exposure to diagnostic ultrasound for 3-21 min showed no harm to the fetal cells. The data obtained in our research support the hypothesis that diagnostic ultrasound is a safe procedure when used for prenatal diagnosis. 


\section{REFERENCES}

Haupt, M., Martin, A.O., Simpson, J.L., lqbal, M.A., Elias, S, Dyer, A., and Sabbagha, R.E. 1981. Ultrasonic induction of sister chromatid exchanges in human lymphocytes. Hum. Genet. 59: 221-226.

Liebeskind, D., Bases, R., Mendez, F., Eiequin, F., and Koenigsberg, M. 1979. Sister chromatid exchanges in human lymphocytes after exposure to diagnostic ultrasound. Science 205: 12731275

Lundberg, M., Jerominski, L., Livingston, G., Kochenour, N., Lee, T., and Fineman, R. 1982. Failure to demonstrate and effect of in vivo diagnostic ultrasound on sister chromatid exchange frequency in amniotic fluid cells. Am. J. Med. Genet. 11:31-35.

Ma, C.-J., Chen, Y.-C., and Huo, P.-D. 1985. Method of amniotic fluid cell culture and preparation of chromosomes. Reproduct. Contracept. (Shanghai) 5(4): 53-54.

Ma, C.-J., Li, Y.-Z., and Sun, Z.-G. 1983. A method for preparation of sister chromatid exchange in human amniotic fluid cells. Reproduct. Contracept. (Shanghai ) 3(2): 54-55.

Morris, S.M. and Palmer C.G. 1978. Effect of ultrasound on human leucocytes sister chromatid exchange analysis. Ultrasound in Med. Biol. 4: 253-258. 